\title{
Coagulation of waste water from the point of view of physic-chemical interactions
}

\author{
Evgeny Alekseev ${ }^{1 *}$, and Svetlana Shambina ${ }^{2}$ \\ ${ }^{1}$ Moscow State University of Civil Engineering, Yaroslavskoe shosse, 26, Moscow, 129337, Russia \\ ${ }^{2}$ Peoples' Friendship University of Russia (RUDN University), 6 Miklukho-Maklaya \\ Street, Moscow, 117198, Russia
}

\begin{abstract}
The widespread use of reagent coagulation in the technology of purification of natural and waste waters is due to the complexity of the chemical composition and phase-dispersed state of the aqua systems of these waters, on the one hand, and the multifactorial action of coagulants on them, on the other. The mechanisms of interaction between coagulants and pollutants of wastewater which are in different phase-dispersed states include the physicochemical phenomena of coagulation, heterocoagulation, adagulation and sorption. The physicochemical concept "coagulation" reflects only one mechanism of coagulants' action on pollutants. This leads to the appearing of the problem in terminological description of the physicochemical processes' combined action when adding coagulants to the water being cleaned. The purpose of the study is to justify the use of universal indicators characterizing the multifactorial nature of the coagulation process, its formalization and quantitative assessment. The solution of the problem is to use the concept of "coasorption", which determines the multifactorial nature of the interaction between coagulants and pollutants, and to use the technological indicator "specific coasorption", which establishes a quantitative relationship between the pollutants' indicators and the dose of coagulant. The test coagulation's results of two different types of synthetic dyes' solutions using aluminum salts reflect the difference in the mechanisms of their interaction with the coagulant. The formula for coasorption isotherm can be used to determine the required doses of reagents for coagulation of water basing on the initial and residual content of pollutants in the purified waters.
\end{abstract}

\section{Introduction}

The method of reagent coagulation in water purification technology has become widespread when solving problems of water supply to populated areas and industrial enterprises. Reagent coagulation technologies are used for solving a significant part of the problems on protecting water objects from pollution with wastewater [1-3]. This proliferation of coagulation methods is due to the complexity of the chemical composition and phasedisperse state of aquatic systems, on the one hand, and the multifactorial action of coagulants on them, on the other.

\footnotetext{
* Corresponding author: AlekseevE@mgsu.ru
} 
The coagulation method has historically evolved from the experience of natural water purification. Indicators of "color" and "turbidity" in the water conditioning technology for domestic and drinking purposes have traditionally been a priority. Both of these indicators, in most cases, are due to the presence of colloidal systems of organic and mineral origin in natural waters. When colloids interact with hydrolysis products of metal salts, then the phenomenon of coagulation occurs in the strict physicochemical sense. The result of this is that the physicochemical concept of "coagulation" has taken root as a synonym for the method of water purification by coagulation. This approach is acceptable for colloidal contaminants.

In accordance with the physicochemical factors of the aquatic systems' stability, the coagulation technology is used to disrupt the aggregate and sedimentation stability of the pollutants' poly-disperse systems [4]. In many studies on wastewater treatment processes, a multifactorial mechanism of interaction between hydrolysis products of mineral coagulants and the pollutants has been noted [5-7]. A complex of physicochemical phenomena leads to a violation of the aggregative and sedimentation stability of wastewater aqua systems when coagulants are added $[8,9]$.

Along with the "classical" mechanism of coagulation of colloidal substances, one of the possible mechanisms explaining the violation of the aggregate stability of dispersed wastewater systems when using mineral coagulants is heterocoagulation [10]. A disperse system with an opposite charge is formed $n$ the process of hydrolysis of the coagulant salts in purified water. This system consists of micro particles of poorly soluble metal hydroxides. This contributes to the convergence of dispersed particles of pollutants and metal hydroxides. As a result of mutual neutralization of the dispersed systems' charges, they lose their aggregate stability and in the future can be effectively isolated from water by using separating processes [11].

When mixing micro dispersions of different nature, the coagulation phenomena of one or more aggregatively stable systems can also be observed as a result of adagulation (heterogeneous adhesive coagulation). According to S.S. Voyutsky, one of the reasons for the loss of stability of dispersed systems in the presence of a foreign surface is the adsorption of the stabilizer on this surface and decrease in its concentration in the colloidal system [12]. The role of such a stabilizer in wastewater is often played by the surfactants which present in it.

Currently, there is no technological concept that would reflect the variety of mechanisms of coagulants' and pollutants' interaction. With the widespread use of the coagulation method in the technology of natural and waste waters purification, the physicochemical concept of "coagulation" replaces a whole complex of physical, chemical and physicochemical interactions of metal salts (coagulants) with a complex polydisperse heterophase system of pollutants in water. This is one part of the problem.

Another part of the problem is a technological one. It consists in the absence of a formalized description of the combined action of physicochemical processes in the coagulation of water and their quantitative indicator.

Traditionally, the indicators "coagulant dose" and "specific coagulant dose" are used in the technology of water purification by reagent coagulation [13].

The coagulant dose is preliminarily determined by the "trial coagulation" method of a specific water sample according to the best result. Evaluation of the result, as a rule, is carried out according to the graphical dependence of the form:

$$
C_{f}=f\left(D_{c}\right)
$$

where $C_{f}$ is the final content of the pollutant, $m g / l ; D_{c}$ is the dose of coagulant, $m g / l$.

The second indicator is the "specific dose of coagulant". This is the mass of the reagent, referred to the initial content of specific types of pollutants. It is focused on the extraction 
of certain groups of pollutants and does not have a deterministic relationship with the result of water purification.

These indicators do not reflect the essence of the processes of removing pollutants and are not directly correlated with the result of coagulation. Therefore, the analytical determination of the required dose of coagulant for other values of the initial and final content of pollutants is actually impracticable.

An integral indicator is required for the technological analysis of water purification processes by coagulation, for engineering and technological calculations of treatment complexes, for assessing economic profitability and solving many other problems. That indicator connects the amount of the introduced coagulant with the result of its use, regardless of the types of interactions with pollutants.

The results of processing a large number of experimental data on the use of coagulants for wastewater treatment confirm the fundamental possibility of applying methods of formalizing indicators to the coagulation process by analogy with the sorption process.

To describe and calculate the sorption process, such technological concepts and indicators as "sorption isotherm", "equilibrium concentration", and "specific sorption" are traditionally used. These concepts and indicators are universal both in relation to the extracted substances and to the types of sorbents. In addition, by the graphical appearance of the sorption isotherms, one can judge the mechanisms of interaction between the sorbent and sorbtiv. With regard to coagulation, a problem arises with terminological definitions. Sorption is the main type of interaction between sorbents and sorbtivs. But during the coagulation of wastewater, "coagulation" is not the main type. Moreover coagulation itself may not exist at all in a complex system of interactions. The indicator "specific sorption" is accepted and widely used in technological processes of sorption. This indicator does not contradict the essence of the physicochemical interaction. A similar indicator in relation to the coagulation method ("specific coagulation") is nonsense from the standpoint of the fundamental foundations of physical chemistry.

The purpose of the work was to substantiate the use of universal indicators characterizing the multifactorial nature of the coagulation process and their formalization. This would make it possible to calculate the coagulation process for all types of wastewater.

\section{Methods}

The solution to the problem of terminological definition for many of processes of coagulants' action on aqua systems is to use the technological concept of "coasorption" (coagulation - sorption). The proposed concept reflects the prevailing complex of coagulation-sorption processes of coagulants' interaction with pollutants as a generalized result of the coagulation technology's application. The "specific coasorption" indicator is based on the concept of coasorption. It can be defined as the ratio of the "removed" value of the estimated indicator to the coagulant dose by metal ion or by dry mass when a stable (equilibrium) value of the pollutant indicator value in purified water is reached [14]:

$$
S=\left(C_{0}-C_{\mathrm{K}}\right) / D_{\mathrm{c}}
$$

where $S$ is the specific coasorption, $g / g ; C_{o}$ and $C_{f}$ are the initial and final contents of the pollutant, respectively, $m g / l ; D_{c}$ is the coagulant dose, $m g / l$.

Accordingly, the graphic interpretation of the specific coasorption in the form of an isotherm can be represented by a functional dependence of the following form:

$$
S_{e}=f\left(C_{\mathrm{e}}\right)
$$

where $S_{e}$ is the value of specific coasorption at the equilibrium concentration of the pollutant, $\mathrm{mg} / \mathrm{mg}$; $C_{e}$ - the corresponding value of the equilibrium concentration, $\mathrm{mg} / \mathrm{l}$. 
To substantiate the expediency of using the proposed indicators, it was necessary to obtain the results of a trial coagulation for solutions of organic compounds with different chemical structures.

Two types of organic dyes were chosen for the experiments:

- acidic: "chrome brown" and "blue 74";

- active: "bright blue $\mathrm{KX}$ " and "bright red 5CX".

A solution of aluminum sulfate $\mathrm{Al}_{2}\left(\mathrm{SO}_{4}\right)_{3} \cdot 18 \mathrm{H}_{2} \mathrm{O}$ was used as a coagulant.

\section{Results}

Figure 1 shows the results of trial coagulation in the form of dependence (1) for two different types of synthetic dyes when coagulated with aluminum salts.
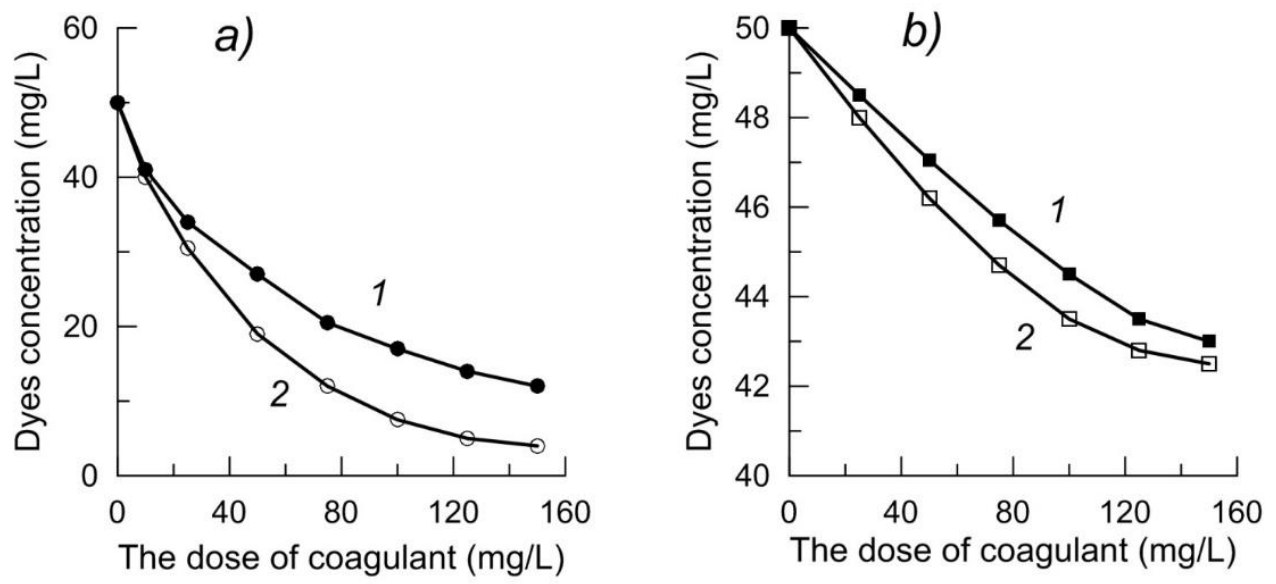

Fig. 1. Coagulation of dyes: $a$-acidic: 1 - chrome brown; 2- blue $74 ; b$-active: 1 - bright blue CC; 2 bright red 5CX.

The results of trial coagulation obtained in this experiment were processed using equation (2) and are presented in Figure 2 in the form of coasorption isotherms in accordance with dependence (3).

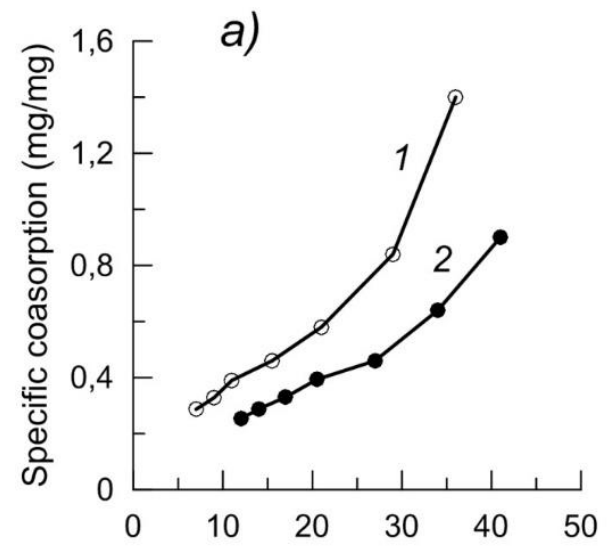

Equilibrium concentration $(\mathrm{mg} / \mathrm{L})$

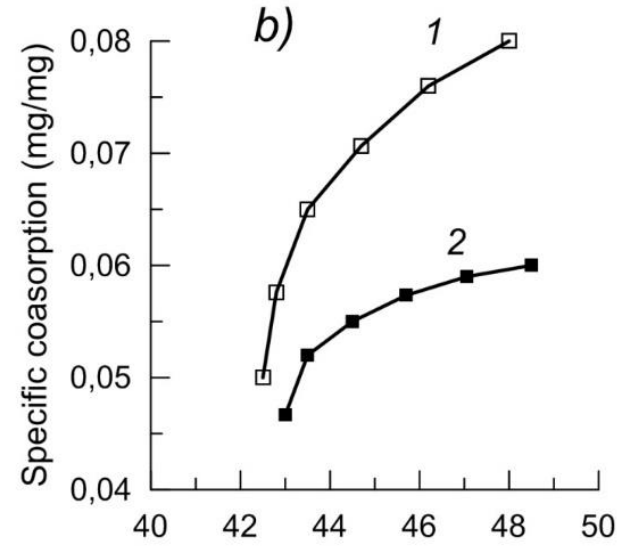

Equilibrium concentration $(\mathrm{mg} / \mathrm{L})$

Fig. 2. Isotherms of dyes' coasorption: $a$ - acidic: 1 - blue 74; 2 - chrome brown; $b$-active: $1-$ bright red 5CX; 2 - bright blue $\mathrm{KX}$. 


\section{Discussion}

The nature of the curves in Figure 1 shows that with an increase in the dose of coagulant, the content of dyes decreases. It can also be seen that the active dyes are extracted less efficiently.

Based on the dependences of the trial coagulation, it is possible to determine the value of the coagulant dose to achieve the required result only for a given initial concentration of dyes. It is not possible to calculate the required dose of coagulant for a different value of the initial concentration of the extracted substance. At the same time, it is also impossible to establish the mechanism of pollutants' interaction with a coagulant by the shape of the curves.

The specific coasorption dependences (Fig. 2) reflect much more information on the nature of the pollutants' interaction with coagulants. First of all, it is necessary to note the significant differences in the shape of the coasorption isotherms curves for acidic and active dyes. This difference in the amount of dyes coasorption is due to different mechanisms for the removal of pollutants.

Analysis of the shape of the coasorption isotherms curves allows to establish the type of interaction between coagulants and pollutants. Thus, acid dyes (see Fig. 2 a) in the zone of low concentrations are extracted during coagulation by the adsorption mechanism; this is reflected in the convex part of the curve. As their content increases, a violation of the aggregate stability of dyes begins to manifest itself as a result of the coagulation phenomenon, which causes the transition of the form of coasorption isotherm from convex to concave. When the content of acid dyes is more than $25-30 \mathrm{mg} / \mathrm{l}$, the interaction with the coagulant is completely dependent on the coagulation mechanism. This is confirmed by the value of specific coasorption of more than $1.0 \mathrm{mg} / \mathrm{mg}$, since the excess of the mass of the extracted substances relative to the mass of the reagents is possible only if the aggregate state of the aqua system is disturbed as a result of its coagulation.

The convex character of the coasorption isotherms reflects the adsorption mechanism of the extraction of limitedly associated structures (see Fig. 2b). So ions of active dyes, which have a low ability to associate, are being extracted during coagulation very insignificantly. The value of the specific coasorption of these substances varies in the range of $0.05-0.08$ $\mathrm{mg} / \mathrm{mg}$. This is due to the phenomena of these substances' sorption on the accessible surface of aluminum hydroxide floccules.

The considered examples of coagulation of organic substances solutions with different chemical structures indicate the possibility of using the "specific coasorption" indicator and its graphic interpretation in the form of an isotherm as an indicator option for analyzing the components of the water purification process.

The "specific coasorption" indicator is also of practical importance. It allows, on the basis of the approximation of the empirical dependence (3), to calculate the required doses of coagulant according to the indicators of pollutants in wastewater before coagulation and in purified water according to equation (4):

$$
D_{c}=\left(C_{0}-C_{f}\right) / S_{c}
$$

where $D_{c}$ is the dose of coagulant, $m g / l ; C_{0}$ and $C_{f}$ are the initial and final content of the pollutant, respectively, $\mathrm{mg} / \mathrm{l} ; S_{f}$ - specific coasorption corresponding to the final content of the pollutant, $m g / m g$, which was found by the approximating equation.

In addition, equation (4) can be used to calculate the doses of reagents as applied to fractional coagulation technology. 


\section{Conclusions}

The physical and chemical mechanisms of the interaction of pollutants with coagulants during the treatment of industrial wastewater are a complex and multifactorial system. Along with the coagulation of colloidal substances, the resulting dispersion of metal hydroxides, due to the developed surface, has a high adsorption capacity for many types of wastewater pollutants. In addition, the interaction reactions of acid residues and other anions at the stage of hydrolysis of coagulant salts, changes in $\mathrm{pH}$ and mineralization can lead to a violation of the aggregate stability of the wastewater aqua system with the formation of a new dispersed phase. A part of dispersed pollutants can be involved in the sedimentation process as a result of hydro-mechanical interactions with floccules of metal hydroxides. In this case, the impurities that were not involved in the current processes of coagulation and sorption can be removed.

The concept of "coasorption" brings together all the factors that lead to a decrease in the content of pollutants in water when using coagulants. The generalizing indicator of coagulation "specific coasorption" establishes a functional relationship between the dose of coagulant and water pollution before and after its purification. The visualization of the indicator in the form of functional dependencies of the specific coasorption isotherms is of great scientific and practical importance, since it allows one to "reveal" the mechanisms of coagulants' interaction with pollutants and to evaluate the efficiency of the reagents based usage on the overall result.

Technological calculations performed using the approximating equations of the specific coasorption isotherms ensure the determination of the required doses of reagents when coagulating water according to the specified values of the content of pollutants in the source and purified water.

\section{References}

1. S. Bouranene, N. Attia, P. Fievet, Filtr. Sep. 42-45 (2015)

2. M. Verma, R.N. Kumar, Persp. Sc. 8 492-494 (2016)

3. J. Lin, S.J. Couperthwaite, G.J. Millar, Sep. Purific. Tech. 177 207-222 (2017)

4. A.K. Zapolsky, Water purification by coagulation (Kamyanets-Podolsky: "Medobory 2006" 2011)

5. S.V. Getmantsev, I.A. Nechaev, L.V. Gandurina, Treatment of industrial waste water with coagulants and flocculants (Moscow: ASV Publishing House 2008)

6. E.E. Goncharenko, B.S. Ksenofontov, A.M. Golubev, Study of the stability and coagulation of lyophobic sols using computer technology 1 54-65 (2014)

7. B. Wang, Y. Shui, M. He, P. Liu, Biochem. Eng. J. 121 107-117 (2017)

8. V.L. Draginsky, L.P. Alekseeva, S.V. Getmantsev Coagulation in natural water purification technology (Moscow: Publishing house Nauka 2005)

9. V.P. Nebera, Flocculation of mineral suspensions (Moscow: Publishing house Nedra 2006)

10. B.V. Deryagin, Coll. J. 16 (6) 425-438 (1954)

11. G. Sontag K. Strenge, Coagulation and stability of dispersed systems (Leningrad: Publishing house Chemistry 2003)

12. S.S. Voyutsky, Colloidal chemistry course (Moscow: Publishing house Chemistry 1989)

13. E.D. Babenkov, Water purification with coagulants (Moscow: Publishing house Nauka, 1977)

14. E.V. Alekseev, Revista de la Universidad Del Zulia. $3^{\text {a }}$ Época. Año 1130 162-177 (2020) 